
comunidad académica universitaria y las pymes, distrito Barranquilla. Económicas CUC, 37(1), 123-134. DOI: http://dx.doi.org/10.17981/econcuc.37.1.2016.06

\title{
Consultorio contable: enlace entre la comunidad académica universitaria y las Pymes, Distrito Barranquilla ${ }^{1}$
}

DOI: http://dx.doi.org/10.17981/econcuc.37.1.2016.06

Alexander Ruz Gomez ${ }^{2}$

\section{Resumen}

En el transcurso de la vida productiva de la empresa se comienzan a presentar a los propietarios del negocio una serie de necesidades de tipo administrativa, organizativa y contable. En función de ello, se estudia lograr el fortalecimiento del enlace entre la comunidad académica universitaria y las pymes, por medio del diseño e implementación de un consultorio contable con el fin de mejorar las condiciones administrativas-financieras de las PYMES de la ciudad de Barranquilla. Su desarrollo parte de una investigación descriptiva, Diseño no experimental corte transversal. La técnica seleccionada fue la encuesta a través del diseño de un instrumento de tipo dicotómico-mixto, aplicado a los gerentes de estas empresas. Los resultados determinan que las pequeñas y medianas empresas se han convertido en el eje principal del crecimiento de la economía Colombiana. La tarea de las instituciones de educación superior debe estar dirigida no sólo a transmitir conocimientos, sino que también se debe orientar a que su labor se extienda más allá de la experiencia de la teoría en las aulas, esto hace referencia al enlace que debe existir entre dichas instituciones y las pymes.

Palabras clave: consultorio contable; PYMES; comunidad académica universitaria; Distrito Barranquilla.

Recibido: 22.1.2016 Devuelto para revisión: 1.3.2016 Aceptado: 5.4.2016

\begin{abstract}
${ }^{1}$ Artículo científico derivado de la investigación "Consultorio Contable: Enlace Entre La Comunidad Académica Universitaria y Las Pymes de la ciudad de Barranquilla-Colombia”, financiada por la Simón Bolívar, Barranquilla, Colombia.

${ }^{2}$ Doctorando en Ciencias Gerenciales. Universidad Rafael Belloso Chacín-Venezuela. Magister en Gerencia Estratégica. Universidad Rafael Belloso Chacín-Venezuela. Especialista en Gerencia estratégica de mercadeo. Universidad Nacional Abierta Y A Distancia (Unad) - Pasto, Colombia. Contador Público. Universidad de la Guajira- Colombia. Docente investigador adscrito al grupo de investigación pensamiento contable. Universidad Simón Bolívar. aruz@unisimonbolivar.edu.co
\end{abstract}

- The author; licensee Universidad de la Costa - CUC

Económicas CUC vol. 37 no. 1, pp. 123-134. Enero - Junio, 2016 Barranquilla. ISSN 0120-3932 Impreso, ISSN 2382-3860 Online

(ㄷ) $(1) \Theta \Theta$ 


\title{
Accounting Office: link Between the Academic Community College and Smes. Barranquilla-Colombia
}

\begin{abstract}
During the productive life of the company, a series of administrative, organizational and accounting needs begin to be presented to business owners. According to it, is studied the strengthening of the link between the university academic community and SMEs, through the design and implementation of an accounting office in order to improve the administrative and financial conditions of SMEs in the city of Barranquilla. Its development starts from a descriptive research, non-experimental cross-sectional design. The selected technique was the survey through the design of a dichotomous-mixed type instrument, applied to the managers of these companies. The results determine that small and medium enterprises have become the main axis of the growth of the Colombian economy. The task of higher education institutions should be directed not only to transmit knowledge, but also should be directed to extend their work beyond the experience of the theory in the classroom, referring to the contact or in this case, a link that must exist between these institutions and SMEs.
\end{abstract}

Keywords: accounting practice; SMEs, university academic community; Barranquilla city.

\section{Introducción}

Como complemento al conocimiento aprendido en las aulas, los estudiantes tienen la necesidad de vivir o experimentar casos cotidianos presentados en el mundo empresarial a nivel de consultoría y a nivel operativo. Así, el consultorio contable es el espacio propicio para la puesta en práctica de lo aprendido; es en esencia el espacio para corroborar lo teórico con la realidad empresarial y ponerse a prueba, entrando en contacto, por medio de estas actividades, con el entorno que los rodea (Mercado y Palmerín, 2005).

Es por esto que existen en un gran número de instituciones educativas en todo el país y la región los consultorios contables, como respuesta a la necesidad de articulación de la academia y el mundo empresarial. No obstante, existe la necesidad de que la comunidad académica esté en contacto directo con el mundo empresa- 
rial, tejiendo cierta relación que beneficie de una $u$ otra forma a los dos actores que entraran en contacto.

Igualmente, los consultorios contables permiten apoyar y fortalecer algunos procesos en las instituciones educativas desde la facultad, una vez puestos en marcha, esto por medio de equipos conformados por estudiantes y docentes de la facultad, quienes realizan asesorías empresariales, trabajos de investigación, desarrollo de prácticas contables y apoyo a la creación de empresas que beneficien a la región (Cleri, 2007).

En Colombia, las organizaciones están frente a mercados que a diario experimentan cambios, haciendo referencia a tratados de libre comercio, acuerdos bilaterales, la alta competitividad que presentan otras empresas en el mercado, las nuevas tecnologías, los novedosos productos y servicios, entre otros. Las empresas pueden prepararse para todos estos retos adoptando $\mathrm{y}$ adaptando posiciones que les permitan superar los cambios en el entorno (Mercado y Palmerín, 2005).

Las pequeñas y medianas empresas son un elemento fundamental en la generación de empleo y en el crecimiento económico de un país, lo que demuestra que existe una estrecha relación entre el grado de desa- rrollo de las pymes y el nivel del ingreso per cápita del Estado. Ésta, entre otras razones, justifica la preocupación permanente del sector privado y de las autoridades económicas por impulsar su crecimiento y consolidación (Arenas, Charris, Manga y Ríos, 2010).

La carencia de asesoría organizativa y contable desde el inicio de sus operaciones, donde el empresario debe tener claridad frente a las obligaciones fundadas en su actividad económica, es una de las principales causales de disolución y del poco rendimiento económico esperado por los empresarios. Una correcta asesoría y acompañamiento permanente reduciría de manera sustancial el número de empresas con dificultades de orden administrativo y fiscal que deciden cerrar sus puertas.

Es así como todo este proceso busca lograr la competitividad interna y externa, como producto del compromiso y pertinencia de la gerencia de la organización y con base en la información oportuna. Este es un proceso de doble vía que tiene que generar credibilidad y confiabilidad entre todos los integrantes de la empresa u organización.

Por lo anterior, en el ámbito empresarial, es claro el papel que desempeña la gerencia al interior de las empresas debido a que 
ésta orientará de mejor manera los procesos y ayudará a que los procesos organizacionales se potencialicen y arrojen sus frutos en pro de la organización, dejando de lado los viejos problemas de tipo organizacional, desarrollo tecnológico y productivo.

Uno de los principales problemas que tienen las pymes es que presentan un nivel bajo de ingreso, razón por la cual no cuentan con una estabilidad económica que les permita sostenerse en su actividad comercial. A lo que se suma que las condiciones de acceso al crédito son poco competitivas internacionalmente en todos los países de la región por causa de los altos costos de crédito con los que cuentan estas empresas, además, los plazos ofertados son cortos, y las exigencias, elevadas en garantías. Interviene también, y es muy común, la falta de confianza de los bancos respecto a los proyectos de estas empresas. Aunque se sabe que la razón principal de la severidad de estas condiciones suele justificarse por la gran cantidad de pymes que se generan, que a diferencia de las grandes empresas, es muy elevada (Arenas et al., 2010).

Pero no sólo es por la falta de apoyo económico, también interviene el deficiente control que presentan la mayoría de estas empresas; las más comunes, son la falta de transparencia contable y el costo de obtención de la información adecuada. El poco conocimiento de los sistemas básicos de control de gestión da lugar a una administración que presenta fraudes, desconocimiento del verdadero estado financiero de la empresa, gastos innecesarios y mal manejo de los inventarios.

En segunda instancia es importante hacer referencia a otros grandes problemas que presentan las pymes como lo son la normatividad vigente, la falta de independencia jurídica de influencias políticas o grupos poderosos, la falta de seguridad jurídica de los contratos y las deficiencias en las leyes, lo cual influye en gran parte en su desarrollo. El poco desarrollo informático, tecnológico y productivo también juega un papel fundamental en las falencias de estas empresas, lo cual implica que la información contable se presente de una manera desactualizada y se origine un difícil manejo.

Por último, se debe resaltar que es necesario que la contabilidad de las pymes se lleve de acuerdo con los principios y normas que establece la ley; llevar la información contable de una forma organizada, sistematizada y fidedigna, con el fin de identificar, interpretar, analizar, evaluar e informar todas las operaciones que se llevan a cabo de una manera más eficiente y rápida. 
Las pequeñas y medianas empresas se han convertido en el eje principal del crecimiento de la economía colombiana debido a que con el tiempo se ha identificado como soporte del desarrollo empresarial.

No obstante, sus dificultades en los procesos de gestión, obtención de recursos e informalidad obstaculizan el crecimiento de las mismas. Según el expresidente de la Asociación Colombiana de la Micro, Pequeña y Mediana Empresa - ACOPI, Juan Pinto Saavedra (2005): "las dificultades o retos vitales que enfrentan las pymes son: la limitación en oferta y recursos, la baja competitividad en gestión, la falta de orientación al mercado y la falta de cultura innovadora". De acuerdo con lo mencionado, se puede inferir que las pymes enfrentan situaciones en distintos frentes que las limitan en sus actuaciones en cada una de las áreas de la gestión organizacional.

\section{Tipo de investigación}

La presente investigación fue catalogada como descriptiva, debido a que se investigó a un grupo de personas (gerentes) quienes describieron las características resaltantes de una determinada situación o problema, considerando que los objetivos de la misma ayudaron al análisis para el pre- sente estudio; al mismo tiempo se tipifico como transversal, por cuanto las variables consultorio contable y pymes se medirán una sola vez a través del cuestionario diseñado, dirigido a los gerentes de las empresas. Según Hernández, Fernández y Baptista (2003), los estudios descriptivos buscan especificar las propiedades, las características y los perfiles de las personas, grupos, comunidades, procesos, objetos o cualquier otro fenómeno que sea sometido a un análisis. Es decir miden, evalúan o recolectan datos sobre diversos conceptos (variables).

Por otra parte, Arias (2006) indica que la investigación descriptiva consiste en la caracterización de un hecho, fenómeno, individuo o grupo, con el fin de establecer su estructura o comportamiento. Los resultados de este tipo de investigación se ubican en un nivel intermedio en cuanto a la profundidad de los conocimientos se refiere.

\section{Diseño de la investigación}

En atención al diseño, éste se clasifica como documental, de campo y no experimental. Además, la estrategia de investigación estuvo definida por el origen de los datos y por la manipulación o no de las condiciones en las cuales se realiza el estudio. Arias (2006) señala que los datos 
de interés se recogen en forma directa a la realidad mediante el trabajo concreto del investigador y su equipo.

Este estudio se sitúa entre los diseños no experimentales y transaccionales descriptivos, cuyo objetivo se ajusta al consultorio contable como enlace entre la comunidad universitaria y las pymes, describiendo sus beneficios sin perturbar el entorno en un momento determinado.

La investigación no experimental (Arias, 2006) la definen como la investigación que se realiza sin manipular deliberadamente variables, es decir, se trata de estudios donde no se hace variar en forma intencional las variables independientes para ver su efecto sobre otras variables.

La población de esta investigación estuvo constituida por un grupo conformado por los gerentes de las pymes en la ciudad de Barranquilla. Este grupo suma 171 empresas.

Según Arias (2006), la población es el conjunto finito o infinito de elementos con características comunes para los cuales serán extensivas las conclusiones de la investigación. Ésta queda delimitada por el problema y por los objetivos del estudio.
Esta población se tipifica como finita; según lo reseña Méndez (2008), este tipo de universos son iguales o inferiores a cien mil (100.000) unidades. Asimismo, la población se cataloga como accesible, por cuanto al ser reducida en tamaño y estar ubicada en un área geográfica con posibilidades de ser abordada por el investigador, será factible realizar el presente estudio sobre la totalidad de los sujetos.

La validez del instrumento se obtuvo mediante al juicio de un (1) experto en el área, quien valoró y emitió un concepto acerca de la pertinencia de cada ítem elaborado con respecto a los objetivos de la investigación, las variables, las dimensiones y los indicadores.

Para determinar la confiabilidad del cuestionario elaborado, se procedió a aplicar una prueba piloto a una población con características similares a las del presente estudio que no forman parte de la misma. Las respuestas de los mismos permitieron determinar un coeficiente de confiabilidad, para lo cual se utilizó la técnica de Kuder-Richardson, aplicándose la fórmula del método antes descrito:

$$
\text { Donde: } r=\frac{k}{k-1}\left(1-\sum \frac{\sigma^{2}}{\sigma^{2}}\right)
$$


$\mathrm{r}=$

coeficiente de confiabilidad KuderRichardson.

$\mathrm{k}=\quad$ Número de ítems

$\Sigma \mathrm{o}^{2}=$ Sumatoria de la varianza de los ítems.

$\hat{o}^{2}=$ Varianza total.

Para las variables consultorio contable y pymes se obtuvo un coeficiente $\mathrm{r}=0.39$, lo cual es indicativo de que el instrumento es medianamente confiable, según lo referido por Hernández y otros (2006), quienes establecen la siguiente escala:

1. Der $=0.01$ a $r=0.33$ es baja confiabilidad;

2. De $\mathrm{r}=0.34$ a $\mathrm{r}=0.67$ es medianamente confiable; y

3. De $\mathrm{r}=0.68$ a $\mathrm{r}=1$ es alta confiabilidad.

Se analizan y discuten los resultados obtenidos del proceso de recolección de la información con la aplicación del instrumento diseñado para la presente investigación denominada Consultorio contable: enlace entre la comunidad académica universitaria y las pymes de la ciudad de Barranquilla. Estos son expuestos siguiendo el orden de presentación de las dimensiones desarrolladas para las variables consultorio contable y pymes.

\section{Análisis de los resultados}

Variable: pymes

Dimensión: relación de cooperación.

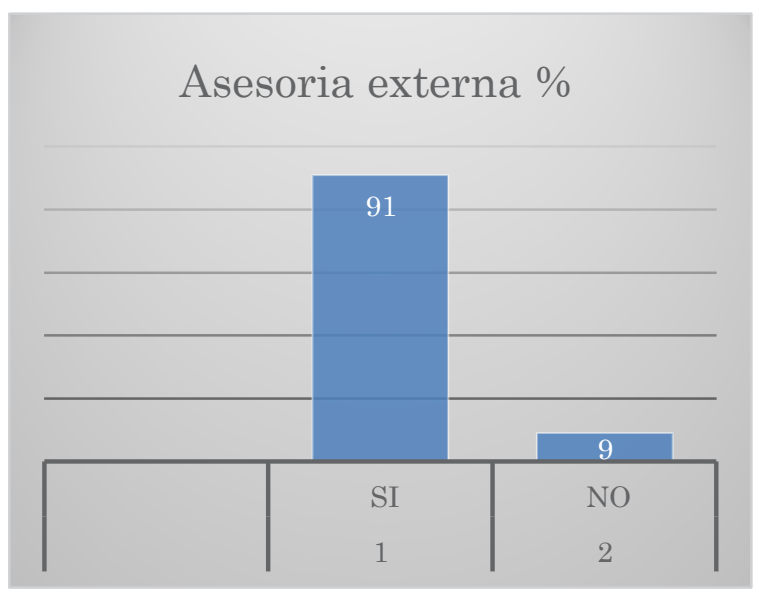

Figura 1. Indicador: asesoría externa Fuente: el autor (2015).

Los resultados que se reflejan en la figura 1, muestran que en el indicador asesoría externa, un número importante, representado en 156 de los 171 encuestados, respondieron afirmativamente a la pregunta, y sólo 15 contestaron de manera negativa.

Expresado de otra manera, el $91 \%$ de los encuestados dice estar interesado en una asesoría externa y el $9 \%$ dice no estarlo. Es de anotar que la asesoría externa siempre será de gran apoyo para las organiza- 
ciones, sin importar al sector de la economía al que pertenezca. En las organizaciones siempre es bueno contar con un punto de vista diferente al de los colaboradores directos que están en la parte interna de la empresa.

Variable: consultorio contable.

Dimensión: relación de cooperación.

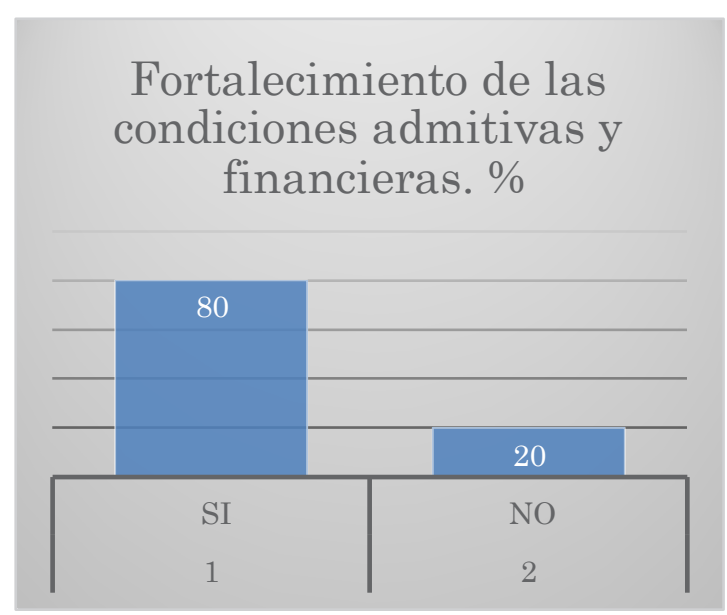

Figura 2. Indicador: fortalecimiento de condiciones administrativas. Fuente: el autor (2015).

Los resultados que se reflejan en la figura 2 , muestran que en el indicador fortalecimiento de condiciones administrativas y financieras, un número importante de sujetos, representado por 127 de los 171 encuestados, respondieron afirmativamente a la pregunta, y 44 contestaron de manera negativa.

Expresado de otra manera, el $80 \%$ de los encuestados considera que su empresa se puede fortalecer con asesoría y acompañamiento externo y el $20 \%$ no lo considera necesario. Es evidente la percepción de los administradores de estas empresas en torno al fortalecimiento de las condiciones administrativas y financieras por medio de una asesoría externa, la cual será brindada por profesionales de diferentes áreas en el marco del desarrollo del consultorio contable.

Variable: consultorio contable

Dimensión: TIC

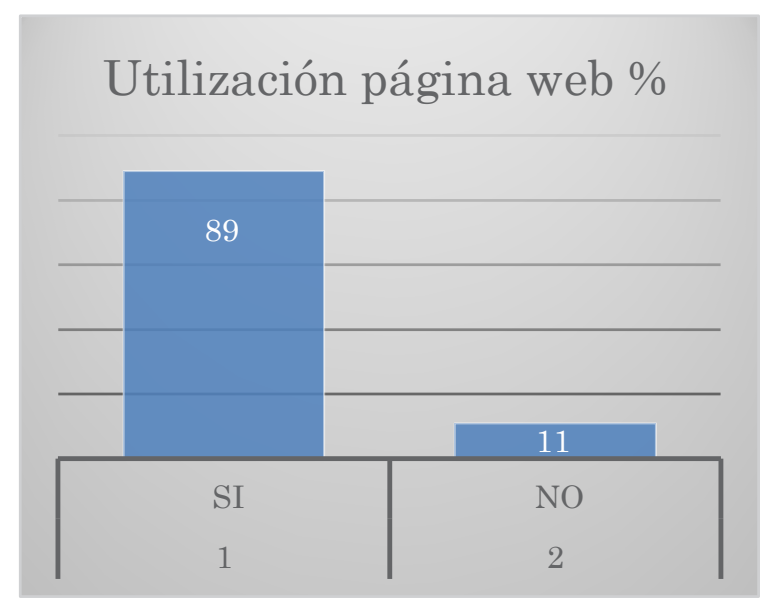

Figura 3. Indicador: Utilidad. Fuente: el autor (2015). 
En los resultados que se reflejan en la figura 3, se observa que en el indicador utilidad, 152 de los 171 sujetos encuestados respondieron afirmativamente, es decir, sí conocen la utilidad de las TIC y 19 de los encuestados respondieron que no conocen la utilidad de las TIC.

Expresado de otra manera, el $89 \%$ de los encuestados dice conocer la utilidad de las TIC, mientras que el $11 \%$ no conoce la utilidad de las TIC. El investigador manifiesta que el conocimiento de la utilidad de las TIC está ligado a la preparación académica y actualización de los gerentes de dichas empresas, puesto que las TIC son herramientas que ayudan a la comunicación y administración de las empresas, lo anterior coincide con lo manifestado por Ca' Zorzi (2011), quien expresa que la introducción de las TIC pone a disposición de la empresa un número inmenso de herramientas que contribuyen a una comunicación más eficiente entre los interlocutores de la empresa, tanto en la comunicación orientada a su personal, como en la orientada al mercado y la que se dirige al entorno social. A pesar de la gran cantidad de posibilidades, podemos agrupar estas herramientas en los siguientes grandes grupos: intranet, extranet, foros, wikis, blogs, entre otros.
Variable: pymes

Dimensión: relación de cooperación

\section{Interes en asesoría \%}

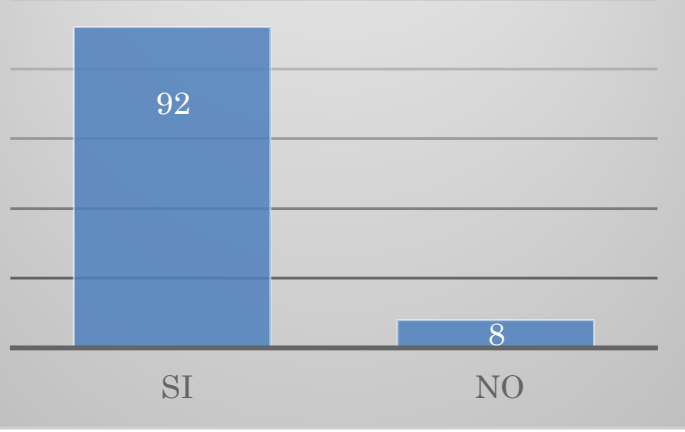

Figura 4. Indicador: interés asesoría externa. Fuente: el autor (2015).

Los resultados que se reflejan en la figura 4 muestran que en el indicador interés asesoría externa, un número importante, representado por 157 de los 171 encuestados, respondieron afirmativamente a la pregunta, y 14 contestaron de manera negativa.

Expresado de otra manera, el $92 \%$ de los encuestados dice estar interesado en una asesoría externa y el $8 \%$ dice no estarlo. 


\section{Variable: pymes}

Dimensión: relación de cooperación

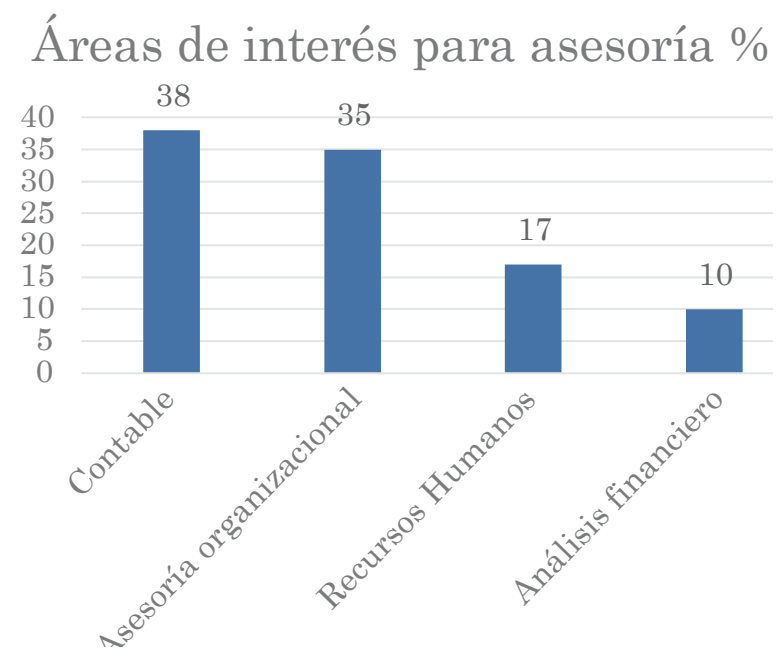

Figura 5. Indicador: áreas de interés. Fuente: el autor (2015).

Los resultados que se reflejan en la figura 5 señalan que en el indicador áreas de interés, un número importante, representado por un $38 \%$ de los encuestados muestran interés por recibir asesoría en el área contable, un $35 \%$ muestran interés por una asesoría organizacional, un $17 \%$ lo hacen para una asesoría en recursos humanos y un $10 \%$ mostró interés en temas de análisis financiero.

Expresado de otra manera, el $100 \%$ de los encuestados dice estar interesado en un área específica para asesoría. Se puede resaltar la necesidad evidente del sector empresarial en cuanto a las áreas en las cuales quisieran recibir asesoría.

Variable: pymes

Dimensión: relación de cooperación

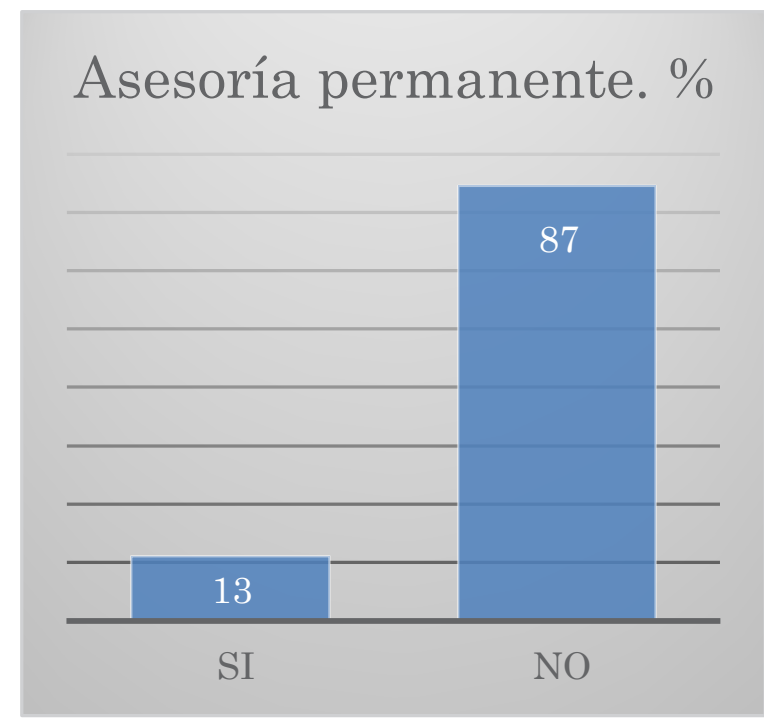

Figura 6. Indicador: asesoría permanente. Fuente: el autor (2015).

En los resultados que se reflejan en la figura 6 , se observa que en el indicador asesoría permanente, sólo 22 de los 171 sujetos encuestados respondieron afirmativamente, es decir, sí cuentan con asesoría permanente en los diferentes temas, y 149 de los sujetos encuestados respondieron que no 
cuentan con asesoría permanente en los temas descritos anteriormente.

Expresado de otra manera, el $13 \%$ de los gerentes encuestados dice contar con asesoría permanente mientras que el $87 \%$ de los sujetos encuestados manifiesta no contar con estas asesorías de carácter permanente.

El investigador manifiesta que el éxito en este tipo de empresas está ligado al conocimiento y habilidades gerenciales que tengan los gerentes en dichas empresas, puesto que a falta de manejo de algunos temas se hace necesaria la asesoría para coadyuvar el proceso gerencial.

\section{Conclusiones}

Cabe aclarar que son pocas las universidades que actualmente tienen consultorios contables en sus instalaciones, se pueden citar la Universidad de la Costa CUC, la Universidad Autónoma del Caribe y la Universidad Libre. Cada una de ellas le proporciona su propio enfoque, funcionalidad y desempeño, así como su propia organización. Pero cuyo fin último es general y es dar atención y asesoría a la comunidad empresarial en diferentes frentes, como el administrativo, el contable y el tributario, entre otros.
Son muchas las necesidades apremiantes que cada organización o empresa tiene, sin importar el sector de la economía al cual pertenezcan. Es por esto que se estableció que todas las empresas encuestadas tienen distintas necesidades y, con el fin de dar respuesta a éstas, se propuso la creación de un consultorio contable en la Universidad Simón Bolívar, haciendo énfasis en temas contables, organizacionales, de recursos humanos y de análisis financiero.

Por lo anterior, es necesaria la puesta en marcha del consultorio contable al interior de la Universidad Simón Bolívar, para así lograr dar respuesta a todas las necesidades de las pymes y, por ende, crear un enlace entre la comunidad académica universitaria y la comunidad empresarial, logrando una relación de mutuo beneficio para las partes.

\section{Referencias}

Arenas, Y., Charris Y., Manga, J. y Ríos, D. (2010). Deficiencias contables en las pymes del sector comercial ubicada en el centro de Barranquilla. Revista Desarrollo Gerencial. 2(1): 143-166.

Arias, F. (2006). El proyecto de investigación. Introducción a la metodología científica. Caracas: Episteme. 
Ca' Zorzi, A. (2011). Las TIC en el desarrollo de la pymes. Centro internacional de investigaciones para el desarrollo: Algunas experiencias de América Latina. Centro internacional de investigaciones para el desarrollo (IDRC). [En línea]. Recuperado de: www.fomin.org/DesktopModules/EasyDNNNews/DocumentDownload.ashx?port alid $=11 \&$ moduleid $=4488 \&$ articleid $=3$ $42 \&$ documentid $=406$

Cleri, C. (2007). El libro de las pymes. Buenos Aires: Granica.

Hernández, R., Fernández, C. y Baptista, P. (2003). Metodología de la investigación. México: Mc Graw Hill.
Mercado, H. y Palmerín, M. (2005). La internacionalización de las pequeñas y medianas empresas. [En línea]. Recuperado de: http://www.adizesca.com/site/ assets/e-la_internacionalizacion_de_ las_pequenas_y_mediana_empresashmymp.pdf

Méndez, C. (2008). Metodología: Diseño y desarrollo del proceso de investigación, con énfasis en ciencias empresariales. México: Limusa.

Pinto, J. (2005). La Internacionalizacionde la Pyme en Colombia. Recuperado de https:// www.scribd.com/document/52110291/ JuanAlfredoPintoCOLOMBIA-PYMEINTERNACIONAL 